\title{
El Cuestionario de Experiencias Relacionadas con los Videojuegos (CERV): Un instrumento para detectar el uso problemático de videojuegos en adolescentes españoles
}

\author{
The Questionnaire of Experiences Associated with Video \\ games (CERV): An instrument to detect the problematic \\ use of video games in Spanish adolescents
}

\begin{abstract}
Andres Chamarro*,**, Xavier Carbonell***, Josep Maria Manresa**,****, Raquel MunozMiralles $* * * * * * *$, Raguel Ortega-Gonzalez $* * * * * *$, M. Rosa Lopez-Morron********, Carme Batalla-Martinez $* * * * * * * *$, Pere Toran-Monserrat**
\end{abstract}

\begin{abstract}
* Departament Psicologia Basica, Evolutiva i de l'Educacio. Universitat Autonoma de Barcelona, Bellaterra, España. ** Unitat de Suport a la Recerca Metropolitana Nord, IDIAP Jordi Gol, Sabadell, España. *** FPCEE Blanquerna, Universitat Ramon Llull, Barcelona, España. **** Departament d'Infermeria, Universitat Autonoma de Barcelona, Bellaterra, España. ***** CAP Ca n’Oriac, Institut Catala de la Salut, Sabadell, España. ****** CAP Santa Perpetua de Mogoda, Institut Catala de la Salut, Santa Perpetua de Mogoda, España. ******* CAP Castellar, Institut Catala de la Salut, Castellar del Valles, España. ******** CAP Sant Quirze, Institut Catala de la Salut, Sant Quirze del Valles, España.
\end{abstract}

\section{Resumen}

El objetivo del presente estudio es validar el Cuestionario de Experiencias Relacionadas con los Videojuegos (CERV). El cuestionario consta de 17 ítems, desarrollados a partir del CERI de Beranuy y cols., y valora el uso problemático de los videojuegos no masivos. Se ha validado para adolescentes que cursan estudios de secundaria obligatoria. Para la validación se ha realizado un análisis factorial confirmatorio (AFC) y un análisis de consistencia interna. La estructura factorial muestra dos factores a) Dependencia psicológica y uso para la evasión, y b) Consecuencias negativas del uso de videojuegos. Se ofrecen puntos de corte de la escala para sujetos sin problemas en el uso de videojuegos (SP), problemas potenciales en el uso de videojuegos (PP) y problemas severos en el uso de videojuegos (PS). Los resultados indican que se da una mayor prevalencia entre varones y que el uso problemático disminuye con la edad. El CERV parece ser un buen instrumento para el cribado de adolescentes con dificultades derivadas del uso de videojuegos. Estudios futuros deberían relacionar el uso problemático de videojuegos con dificultades en otros ámbitos de la vida, como el académico.

Palabras Clave: adicción a los videojuegos en línea; cuestionarios; estudios de validación; videojuegos; adicción a videojuegos.

\section{Abstract}

The aim of this study is to validate the Video Game-Related Experiences Questionnaire (CERV in Spanish). The questionnaire consists of 17 items, developed from the CERI (Internet-Related Experiences Questionnaire - Beranuy and cols.), and assesses the problematic use of non-massive video games. It was validated for adolescents in Compulsory Secondary Education. To validate the questionnaire, a confirmatory factor analysis (CFA) and an internal consistency analysis were carried out. The factor structure shows two factors: (a) Psychological dependence and use for evasion; and (b) Negative consequences of using video games. Two cut-off points were established for people with no problems in their use of video games $(\mathrm{NP})$, with potential problems in their use of video games (PP), and with serious problems in their use of video games (SP). Results show that there is higher prevalence among males and that problematic use decreases with age. The CERV seems to be a good instrument for the screening of adolescents with difficulties deriving from video game use. Further research should relate problematic video game use with difficulties in other life domains, such as the academic field. Key Words: internet gaming disorder; questionnaires; validation studies; video games; computer games; video game addiction.

Recibido: Febrero 2014; Aceptado: Septiembre 2014

Enviar correspondencia a:

Xavier Carbonell. FPCEE Blanquerna, Universitat Ramon Llull, C/ Císter, 34, 08022 Barcelona, Spain; Email: xaviercs@blanquerna.url.edu 
$\mathrm{E}$ n el mundo desarrollado, los videojuegos son dispositivos electrónicos de ocio muy populares en todas las edades, aunque son los adolescentes los que hacen un uso más intensivo (Lorenci, 2012). A estos juegos se puede acceder desde el ordenador, la videoconsola o el móvil, y jugarse en solitario o con otros compañeros, de forma masiva o múltiple. Actualmente las plataformas más populares son la videoconsola y el ordenador (Connolly, Boyle, MacArthur, Hainey y Boyle, 2012). $\mathrm{Su}$ creciente popularidad ha centrado el interés de padres, investigadores y responsables políticos sobre su potencial dañino (Prot, McDonald, Anderson y Gentile, 2012). De estos videojuegos los que más preocupan son los juegos shooter en primera persona porque se ha descrito que aumentan las emociones, pensamientos y conductas agresivas (Anderson et al., 2010; de la Torre-Luque y Valero-Aguayo, 2013) y que producen una pérdida de sensibilización frente a la agresión (Carnagey, Anderson y Bushman, 2007). De todos los videojuegos, independientemente de que sean tipo shooter, aventura, estrategia, simulación o deporte, preocupa su posible capacidad adictiva (King, Delfabbro y Griffiths, 2013). La prevalencia de problemas relacionados con los videojuegos se ha estimado en el 9\% (Gentile, 2011) y en el 8\% (Porter, Starcevic, Berle y Fenech, 2010)

Aparte del tipo de videojuego, otro aspecto a considerar es cómo se interactúa con el juego. En los videojuegos tradicionales, sea cual sea su género, el jugador juega en solitario contra el programa en una videoconsola u ordenador, si bien, en la actualidad, ambos soportes permiten interacciones en línea. Por otro lado, los Massively Multiplayer Online Role-Playing Games (MMORPG) son una evolución de los juegos de rol y de los videojuegos clásicos. En los MMORPG el jugador crea un personaje o avatar, que aumenta niveles y experiencia en luchas contra otros personajes o realizando diversas aventuras o misiones. Los MMORPG permiten a miles de jugadores interactuar entre ellos en un mundo virtual de forma simultánea porque el mejor modo de progresar es formar grupos o clanes (guilds). Esta última característica es la que los diferencia del resto de videojuegos que pueden jugarse en modo individual o multijugador, porque las consolas modernas permiten la conexión a internet, pero no son masivos. Los MMORPG son juegos de carácter abierto; se pueden escoger las propias rutas de desarrollo argumental y como no existe un final concreto siempre se encuentran nuevos alicientes y actividades a ejecutar. Además son un mundo persistente, el juego prosigue su devenir y evoluciona, cambia y se transforma pese a que el jugador o el clan no estén conectados (Ng y Wiemer-Hastings, 2005). Esta persistencia, a veces, implica una cierta obligación a jugar. En los MMORPG no hay control externo de estímulos y están disponibles las 24 horas del día, siete días a la semana. Ningún juego social es tan accesible (Carbonell et al., 2009).

Recientemente, la quinta edición del Manual Diagnóstico y Estadístico de los Trastornos Mentales, en la sección III, reserva- da a las condiciones que requieren estudio posterior, incluye la adicción a los Massively Multiplayer Online Role-Playing Games (MMORPG) a la que denomina Internet Gaming Disorder (American Psychiatric Association, 2013). Algunos investigadores han detectado que los MMORPG tienen mayores tasas de uso problemático que el resto de videojuegos (Haagsma, Caplan, Peters y Pieterse, 2013; Li y Wang, 2013, Nicolier et al, 2013). La American Psychiatric Association distingue los MMORPG del resto de videojuegos, pero deja una puerta abierta cuando apunta que, en el futuro, la categoría diagnóstica Internet Gaming Disorder puede incluir los non-Internet computerized games, es decir, los videojuegos que se juegan en la consola u ordenador, con o sin conexión a Internet, pero que no son masivos, ni mundos persistentes, ni permiten crear personajes ni son abiertos. Dado que éstos también tienen un cierto potencial adictivo, o cuando menos, afectan negativamente a sus usuarios, parece necesario disponer de información que permita valorar estos riesgos.

En España, se han desarrollado cuatro escalas para evaluar el uso problemático de los videojuegos, una de cribado y tres diagnósticas. Pedrero et al. (2007), basándose en el CAGE de Ewing (1984), construyeron una escala de cribado de ocho trastornos de cuatro ítems por trastorno. Cada uno de estos problemas se exploró mediante cuatro preguntas que reproducen el esquema del CAGE: la autopercepción del problema, la percepción por parte de convivientes, sentimientos de culpa asociados y signos de abstinencia o incapacidad para controlar la conducta. Tejeiro y Bersabé (2002), construyeron el Problem Video Game Playing Questionnaire (PVP), un instrumento unidimensional basada en los criterios DSM IV para la dependencia de substancias y que validaron con una muestra de 223 estudiantes de 13 a 18 años. Entre sus hallazgos más relevantes destacan que los hombres jugaban más y tenían mayor uso problemático que las mujeres y que un grupo de adolescentes tenían problemas con los videojuegos, indicativos de dependencia. Pese al avance que ha supuesto, el PVP presenta algunas limitaciones: a) la naturaleza dicotómica de los ítems, b) las bajas correlaciones de algunos ítems con la escala y, c) la baja consistencia interna. Chóliz y Marco (2011) construyeron el Video Game Dependence Test (TDV). Al igual que el PVP se basa en los criterios DSM IV para la dependencia de substancias. Para validarla utilizaron una muestra de 621 niños y adolescentes entre 10 y 16 años y obtuvieron una consistencia interna de $\alpha=.94$, con 25 ítems agrupados en cuatro factores: Abstinencia, Uso excesivo y tolerancia, Problemas causados por los videojuegos y Pérdida de control. Desde nuestro punto de vista, ambas escalas están limitadas por su construcción en base a una traslación directa de los criterios DSM-IV para la adicción a sustancias a una posible adicción conductual. Recientemente, Labrador, Villadangos, Crespo y Becoña (2013) han desarrollado una medida de uso problemático de nuevas tecnologías (televisión, internet, móvil y videojuegos) pero que no permite un análisis individual del uso problemático de videojuegos. 
Hay que considerar que como en otras TIC, el énfasis en la adicción y el uso problemático no permite visualizar que la alta implicación (engagement) de los usuarios puede no ser patológica (Charlton y Danforth, 2007) e incluso contribuir a conductas saludables, como la socialización (Fúster, Chamarro, Carbonell y Vallerand, 2014). Además, se ha generado una cierta controversia al aducirse que las publicaciones referidas al tema tienen defectos metodológicos, problemas de validez interna y sobreestimación de las consecuencias (Ferguson, 2013; Gunter y Daly, 2012; Valadez y Ferguson, 2012; Kutner y Olson, 2008).

Ahora bien, independientemente de estas consideraciones teóricas, es innegable que se necesita determinar hasta qué punto el uso de videojuegos puede perjudicar el desempeño académico, la relación familiar y la maduración de los adolescentes (Estallo, Masferrer y Aguirre, 2001; León y López, 2003). Además, existe una constante preocupación entre padres y educadores, que solicitan información sobre el diagnóstico y el tratamiento de esta problemática. Por otro lado, la cultura popular refleja la capacidad adictiva de estos juegos tal como se recoge frecuentemente en los medios de comunicación. El colectivo de videojugadores utiliza la expresión ‘viciado' para describir al jugador que ha establecido una relación patológica con uno o más de estos juegos.

Así, teniendo en cuenta las limitaciones de los instrumentos de medida existentes y como continuación de nuestra investigación en la construcción de las escalas para evaluar el uso problemático de internet y móvil (Beranuy, Chamarro, Graner, y Carbonell, 2009), este estudio tiene dos grandes objetivos. Primero, la validación de un cuestionario para medir el uso problemático de videojuegos no masivos y, segundo, determinar el uso problemático de estos videojuegos en los adolescentes españoles. Al igual que en los cuestionarios de uso problemático de internet y móvil (Beranuy et al., 2009), se espera que la estructura factorial del CERV sea bidimensional, con los ítems correspondientes a la preocupación, negación, evasión y deseo de jugar, derivados del CERI, agrupados en un factor, y los de aumento de la tolerancia, reducción de actividades y efectos negativos, derivados del CERM, agrupados en otro factor.

\section{Método}

\section{Participantes}

Las encuestas recogidas forman parte del proyecto JOITIC (Muñoz-Miralles et al., 2013), dirigido a 7168 alumnos de $1^{\circ}$ a $4^{\circ}$ de ESO, de 28 centros escolares públicos y concertados, de la comarca del Vallès Occidental (Barcelona, España), integrados dentro del Programa Salut i Escola de la Generalitat de Catalunya y que aceptaron participar en el estudio. No participaron los centros privados por no estar dentro de dicho programa. Se obtuvieron las respuestas de 5.538 alumnos (77,3\% de participación). La edad media fue de 13,94 años (DT= 1.34), rango entre 12 y 20 años, y el 47,9 \% fueron chicas.

\section{Instrumentos}

El CERV es una versión para videojuegos no masivos de los cuestionarios CERI y CERM (Beranuy et al., 2009). El CERV tiene 17 ítems sobre la preocupación, negación, aumento de la tolerancia, efectos negativos, reducción de actividades, pérdida de control, evasión y deseo de jugar (ver Anexo 1). Los ítems se elaboraron a partir de las subescalas de conflictos inter e intrapersonales del CERI y la de conflictos del CERM. La escala de respuestas es tipo Likert de cuatro puntos.

\section{Procedimiento}

Los datos se recogieron durante el curso académico 201011. Los cuestionarios fueron administrados por los autores del estudio en las aulas escolares. Las autoridades académicas de cada centro, los padres y los alumnos dieron su consentimiento a la administración de los cuestionarios. En la portada del cuadernillo de cuestionarios se explicitaban los objetivos del estudio y el carácter voluntario, confidencial y anónimo de las respuestas. El proyecto fue aceptado por el Comité Ético en Investigación Clínica del Institut Universitari d'Investigació en Atenció Primària (IDIAP) Jordi Gol.

\section{Análisis de datos}

El análisis de datos se realizó con los programas estadísticos SPSS versión 17.0 en español y EQS 6.1 (Bentler \& Wu, 2002). Las técnicas utilizadas fueron: (a) análisis factorial confirmatorio con estimadores robustos, (b) análisis descriptivo, (c) análisis de frabilidad mediante alfa de Cronbach, (d) diferencias de medias mediante t de Student y análisis de varianza con contrastes a posteriori aplicando el test de Bonferroni para comparaciones de medias por pares (de dos en dos). Con el fin de obtener puntos de corte se llevó a cabo un análisis de clusters no jerárquico (K-means) para determinar la existencia de grupos homogéneos de participantes con respecto a las puntuaciones. En el análisis de la estructura interna del cuestionario se realizó un análisis factorial confirmatorio (AFC), utilizando criterios convencionales para estimar el ajuste de la estructura propuesta a los datos. El ajuste se infirió cuando los índices TLI (Tucker Lewis Index) y CFI (Comparative Fit Index) > .95, RMSEA (Root Mean Square Error of Aproximation) $\leq .06$ (Hu y Bentler, 1999; Kline, 2010). De acuerdo con Brown (2006), para comparar los diferentes modelos se utilizó el AIC (Akaike Information Criterion). En este estudio se utilizó la matriz de datos originales para estimar el ajuste del modelo.

\section{Resultados}

\section{Análisis descriptivos}

La media, desviación típica y asimetría y curtosis para cada subescala se puede consultar en la Tabla 2. Las medias son bajas para las dos subescalas, si bien la asimetría y curtosis para la subescala de evasión son muy elevadas. 
Tabla 1

Estadísticos descriptivos y alfa de Crombach para las subescalas del CERV

\begin{tabular}{|c|c|c|c|c|c|c|c|c|}
\hline & N & Media & DT & Min. & Max. & Asimetría & Curtosis & $\alpha$ \\
\hline $\begin{array}{l}\text { Dependencia y } \\
\text { Evasión }\end{array}$ & 4720 & 10,92 & 3,5 & 9 & 36 & 3,09 & 12,3 & 0,869 \\
\hline $\begin{array}{l}\text { Consecuencias } \\
\text { negativas }\end{array}$ & 4815 & 13,5 & 5 & 8 & 32 & 1,01 & 0,7 & 0,861 \\
\hline Total & 4347 & 24,42 & 7,88 & 17 & 68 & 1,7 & 3,72 & 0,912 \\
\hline
\end{tabular}

\section{Análisis factorial confirmatorio}

La estructura de dos factores se sometió a AFC y se comparó con un modelo unidimensional. Las variables fueron consideradas categóricas, por lo que se utilizaron estimadores robustos. La solución unifactorial mostraba un ajuste aceptable a los datos $(\mathrm{S}-\mathrm{B} \chi 2(119)=1354,12 ; \mathrm{p}<0.001$; $\mathrm{AIC}=1118.12 ; \mathrm{CFI}=.96 ; \mathrm{TLI}=.956 ; \mathrm{RMSEA}=.049$ [.047$.051])$. El modelo de dos factores con un factor de segundo orden también presentaba un ajuste aceptable (S-B $\chi 2$ (114) $=835,01 ; \mathrm{p}<0.001 ; \mathrm{AIC}=607.01 ; \mathrm{CFI}=.977 ; \mathrm{TLI}=.973$; RM$\mathrm{SEA}=.038$ [.036-.041]). Dado que el índice AIC es inferior en el segundo modelo se optó por considerar éste como el modelo más plausible. Los dos factores resultantes fueron denominados Dependencia psicológica y evasión y Consecuencias negativas, con 8 y 9 ítems respectivamente. Las cargas factoriales estandarizadas se presentan en la Tabla 2.

Tabla 2

Cargas factoriales estandarizadas

\begin{tabular}{|c|c|c|}
\hline & Evasión & Consecuencias negativas \\
\hline 1 ¿Hasta qué punto te sientes inquieto por temas relacionados con los videojuegos? & $.78^{*}$ & \\
\hline 2. ¿Cuándo te aburres, utilizas los videojuegos como una forma de distracción? & $.77^{*}$ & \\
\hline $\begin{array}{l}\text { 3. ¿Con que frecuencia abandonas las cosas que estás haciendo para estar más } \\
\text { tiempo jugando a los videojuegos? }\end{array}$ & $.84^{*}$ & \\
\hline 8. Cuando tienes problemas, ¿los videojuegos te ayudan a evadirte de ellos? & $.72 *$ & \\
\hline 10. ¿Piensas que la vida sin videojuegos es aburrida y triste? & $.76^{*}$ & \\
\hline $\begin{array}{l}\text { 11. ¿Te enfadas o te irritas cuando alguien te molesta mientras juegas a algún } \\
\text { videojuego? }\end{array}$ & $.76^{*}$ & \\
\hline 15. ¿Quitas importancia al tiempo que has estado utilizando los videojuegos? & $.68^{*}$ & \\
\hline 16. ¿Dejas de salir con los amigos para pasar más tiempo jugando con videojuegos? & $.75^{*}$ & \\
\hline $\begin{array}{l}\text { 4. ¿Te han criticado tus amigos o familiares por invertir demasiado tiempo y dinero } \\
\text { en los videojuegos, o te han dicho que tienes un problema, aunque creas que no es } \\
\text { cierto? }\end{array}$ & & $.76^{*}$ \\
\hline $\begin{array}{l}\text { 5. ¿Has tenido riesgo de perder una relación importante, un trabajo o una } \\
\text { oportunidad académica por el uso de los videojuegos? }\end{array}$ & & $.74^{*}$ \\
\hline $\begin{array}{l}\text { 6. ¿Piensas que tu rendimiento académico se ha visto afectado negativamente por el } \\
\text { uso de los videojuegos? }\end{array}$ & & $.76^{*}$ \\
\hline $\begin{array}{l}\text { 7. ¿Mientes a tus familiares o amigos en lo relativo a la frecuencia y duración del } \\
\text { tiempo que inviertes en los videojuegos? }\end{array}$ & & $.74^{*}$ \\
\hline $\begin{array}{l}\text { 9. ¿Con que frecuencia bloqueas pensamientos molestos sobre tu vida y los } \\
\text { substituyes por pensamientos agradables de los videojuegos? }\end{array}$ & & $.77^{*}$ \\
\hline $\begin{array}{l}\text { 12. ¿Sufres alteraciones de sueño debido a aspectos relacionados con los } \\
\text { videojuegos? }\end{array}$ & & $.77 *$ \\
\hline 13. ¿Cuándo no estás utilizando los videojuegos te sientes agitado o preocupado? & & $.83^{*}$ \\
\hline $\begin{array}{l}\text { 14. ¿Sientes la necesidad de invertir cada vez más tiempo en los videojuegos para } \\
\text { sentirte satisfecho? }\end{array}$ & & $.85^{*}$ \\
\hline 17. ¿Cuándo utilizas los videojuegos, te pasa el tiempo sin darte cuenta? & & $.71^{*}$ \\
\hline
\end{tabular}

Nota: ${ }^{*}=p<.05$ 


\section{Análisis de fiabilidad}

Los coeficientes alfa de Crombach para las subescalas son de 0.869 para la de consecuencias negativas y de 0,861 para la evasión (ver Tabla 2). Todos ellos superan el valor criterio de 0,70 establecido por Nunnally (1978) para establecer una consistencia interna aceptable en el ámbito de la investigación en psicología. La eliminación de cualquiera de los ítems no reportó mejora alguna sobre la fiabilidad de las subescalas, por lo que las dos se mantuvieron íntegras.

\section{Análisis de clusters}

El análisis de clusters ofreció una solución de tres grupos $(\mathrm{F}[2]=10153.8 ; \mathrm{p}<.001)$. El primer grupo $(\mathrm{N}=1154$; $26.8 \%$ ) con las puntuaciones más bajas (entre 17 y 25 puntos) fue categorizada como 'SP' (sin problemas con el uso de los videojuegos). El segundo grupo ( $\mathrm{N}=2885 ; 67 \%)$ con puntuaciones medias (entre 26 y 38 puntos) fue categorizado como 'PP' (problemas potenciales con el uso de los videojuegos). El tercer grupo $(\mathrm{N}=264 ; 6.1 \%)$ con las puntuaciones más elevadas (entre 39 y 68 puntos) fue categorizado como "PS" (problemas severos en el uso de videojuegos). Se observa un mayor porcentaje de chicos en los grupos sin problemas y con problemas frecuentes. Las chicas están más presentes en el grupo de problemas ocasionales (ver Tabla 3). Adicionalmente, se ofrecen baremos en percentiles (ver Tabla 4).

\section{Comparaciones de medias}

Se observan diferencias para el género ( $F[1,4295]$ $\left.=1130.31 ; \mathrm{p}<0.001 ; \eta^{2}=.208\right)$ con mayores puntuaciones para los varones, tanto para la evasión como para las consecuencias negativas (ver Tabla 5). También se observan diferencias significativas para el nivel educativo ( $F[3,4295]$ $\left.=12,20 ; \mathrm{p}<0.001 ; \eta^{2}=.008\right)$ y para la interacción género $\mathrm{x}$ curso $\left(\mathrm{F}[3,4295]=9.84 ; \mathrm{p}<0.001 ; \eta^{2}=.006\right)$. Las comparaciones por pares indican que las diferencias se observan entre los alumnos de $4^{\circ}$ de ESO y el resto, siendo los de $4^{\circ}$ los que muestran hacer un uso menos problemático (ver Tabla 5). Las puntuaciones medias son menores para las niñas, especialmente en el uso de los videojuegos con finalidad de evasión, que es más marcado en el caso de las niñas de $4^{\circ}$ de ESO.

Tabla 3

Categorización de uso problemático de videojuegos en función del género

\begin{tabular}{lcc}
\hline Grado de uso problemático & Masculino & Femenino \\
\hline Sin problemas (SP) & $948(22 \%)$ & $206(4,8 \%)$ \\
Problemas potenciales (PP) & $1028(23,9 \%)$ & $1857(43,2 \%)$ \\
Problemas severos (PS) & $235(5,5 \%)$ & $29(0,7 \%)$ \\
\hline
\end{tabular}

Tabla 4

Percentiles de uso problemático de videojuegos en función del nivel educativo

\begin{tabular}{|c|c|c|c|c|c|c|c|c|c|c|c|c|c|c|c|c|c|}
\hline \multirow[b]{3}{*}{$\begin{array}{c}\text { Curso de } \\
\text { ESO }\end{array}$} & \multicolumn{17}{|c|}{ Puntuación } \\
\hline & \multirow[b]{2}{*}{ Mínimo } & \multirow[b]{2}{*}{ Máximo } & \multicolumn{15}{|c|}{ Percentiles } \\
\hline & & & 3 & 5 & 10 & 20 & 25 & 30 & 40 & 50 & 60 & 70 & 75 & 80 & 90 & 95 & 97 \\
\hline Primero & 17 & 67 & 17 & 17 & 17 & 19 & 20 & 20 & 22 & 23 & 25 & 27 & 28 & 30 & 35 & 40 & 43,5 \\
\hline Segundo & 17 & 65 & 17 & 17 & 17 & 18 & 18 & 19 & 20 & 22 & 24 & 27 & 28 & 30 & 34 & 40,5 & 44 \\
\hline Tercero & 17 & 68 & 17 & 17 & 17 & 18 & 18 & 19 & 21 & 22 & 24 & 26 & 28 & 30 & 36 & 42 & 46 \\
\hline Cuarto & 17 & 65 & 17 & 17 & 17 & 17 & 17,5 & 18 & 19 & 21 & 23 & 25 & 26 & 28 & 33 & 38 & 41 \\
\hline Total & 17 & 68 & 17 & 17 & 17 & 18 & 18,3 & 19 & 21 & 22 & 24 & 26 & 28 & 29 & 35 & 40 & 44 \\
\hline
\end{tabular}

Tabla 5

Medias de uso problemático de videojuegos en función del género y el nivel educativo

\begin{tabular}{ccccc}
\hline \multicolumn{2}{c}{ Masculino } & \multicolumn{2}{c}{ Género } & Femenino \\
\hline Curso de ESO & Evasión & $\begin{array}{c}\text { Consecuencias } \\
\text { negativas }\end{array}$ & Evasión & $\begin{array}{c}\text { Consecuencias } \\
\text { negativas }\end{array}$ \\
\hline Primero & $14.53(4.40)$ & $13.41(4.17)$ & $11.13(3.36)$ & $11.09(2.91)$ \\
Segundo & $14.56(4.54)$ & $13.72(4.37)$ & $10.03(2.50)$ & $10.23(1.88)$ \\
Tercero & $14.69(4.91)$ & $13.70(4.30)$ & $10.16(3.15)$ & $10.35(2.72)$ \\
Cuarto & $13.72(4.41)$ & $12.98(3.79)$ & $9.60(2.72)$ & $10.13(2.81)$ \\
\hline
\end{tabular}




\section{Discusión}

El presente estudio tenía dos objetivos: la validación de una escala para medir el uso problemático de videojuegos no masivos y, segundo, determinar el uso problemático de estos videojuegos en los adolescentes españoles. Nuestros resultados muestran que el CERV es un instrumento con evidencias de validez estructural y buena fiabilidad que parece ser útil para determinar el tipo de uso problemático de videojuegos no masivos.

Niños, adolescentes y adultos utilizan los videojuegos no masivos con finalidad recreativa, como una forma de diversión, de escaparse de la vida cotidiana y relajarse. La estructura factorial validada sugiere que este uso se convierte en problemático cuando este uso saludable genera dependencia psicológica: evasión de los problemas, modificación del estado de ánimo, pérdida de control y focalización. El segundo factor, recoge las consecuencias negativas del uso de los videojuegos: disminución del rendimiento académico, dificultades en las relaciones sociales, conflictos con los adultos, agitación y habituación. Estos dos factores (Dependencia psicológica y evasión y Consecuencias negativas) agrupan los cuatro factores encontrados por Chóliz y Marco (2011), de forma que Dependencia psicológica condensa Abstinencia, Uso excesivo y tolerancia, y Pérdida de control, y que nuestro factor Consecuencias negativas es equivalente al Problemas causados por los videojuegos. La estructura factorial emergente sugiere que el CERV no se ajusta a los criterios del DSM-IV-TR, algo que ya sucedió con el CERI y con el CERM (Beranuy et al. 2009) y cuestiona hasta que punto una mera transposición de los criterios de DSM para substancias es aplicable a una adicción conductual. También podría deberse a que el CERV se centra en la percepción del trastorno.

Al igual que en el estudio de Beranuy et al. (2009) los problemas de uso problemático de los videojuegos parecen vincularse a la interferencia y al desajuste que causan en el estilo de vida, cosa que tiene importantes consecuencias negativas para los adolescentes, que se encuentran en una etapa en que empiezan a construir sus relaciones y formar sus hábitos. Estos resultados son compatibles con los de Lafrenière, Vallerand, Donahue, y Lavigne (2009) que encontraron que los videojugadores que se implicaban de forma obsesiva mostraban experiencias afectivas negativas y conducta problemática. Nuestra medida de uso problemático de videojuegos parece recoger estos dos aspectos del uso del videojuego: la implicación obsesiva y las consecuencias negativas. En todo caso, los dos factores identificados parecen corresponderse con la "saliencia cognitiva y problemas derivados" que caracterizan el uso problemático de Internet (Charlton y Danforth, 2007).

En relación con el segundo objetivo del estudio, nuestros resultados muestran que un $6,1 \%$ de los adolescentes, principalmente chicos, estarían en riesgo de que su uso de los videojuegos tuviera consecuencias negativas. Esta prevalen- cia parece ser consistente con la encontrada por otros investigadores españoles. Así, Oliva (2012) cifró en un 8,42\% los videojugadores adictos, con prevalencia superior en chicos que en chicas. Labrador y Villadangos (2010) obtuvieron prevalencias más bajas, del $0.3 \%$ para chicas y del $2.4 \%$ para chicos, pero consideramos que su medida no recoge toda la complejidad del uso problemático, lo que explicaría la limitada prevalencia.

Por otro lado, ya se ha comentado que no estamos en condiciones de confirmar la existencia de un trastorno adictivo grave y persistente relacionado con los videojuegos u otras tecnologías en base a encuestas poblacionales (Carbonell, Fúster, Chamarro y Oberst, 2012). Esta prudencia se apoya entre otros, en los siguientes factores: a) ausencia de demanda clínica de acorde al porcentaje de usuarios problemáticos identificados en estas investigaciones (aunque se han reportado casos en los servicios de psiquiatría infanto-juveniles); b) los cuestionarios podrían evaluar "preocupación” o uso problemático en lugar de adicción; y c) la normalización de la conducta o ausencia de preocupación a medida que los usuarios crecen. Desde una perspectiva clínica se debe distinguir entre el tiempo perdido durante períodos más o menos prolongados y una verdadera adicción. Estos datos sugieren que los jóvenes son muy sensibles a la presión social y que se perciben a sí mismos como usuarios excesivos de estas tecnologías. Así, es muy difícil utilizar nuestros datos para diagnosticar un trastorno mental de la misma entidad que una dependencia a una sustancia como el alcohol o la cocaína. Sin embargo, de nuestra investigación se desprende que los adolescentes viven con preocupación su relación con los videojuegos no masivos y que este uso merece una atención especial por parte de sanitarios, educadores y familiares.

En todo caso, parece claro que el uso problemático de los videojuegos es un fenómeno típicamente masculino (Labrador et al., 2010; Fuster, Oberst, Griffiths, Carbonell, Chamarro y Talarn, 2012) que se presenta con mayor intensidad en la preadolescencia. La mayoría de los estudios confirman que las percepciones problemáticas son mayores en los más jóvenes pero todavía no podemos determinar si es debido a que su implicación con el ocio electrónico es mayor o porque son conductas que tienden a normalizarse con la maduración. Sabemos que muchos adultos continúan jugando y presumimos que su patrón de uso es menos intensivo del que tenían en la adolescencia pero esta suposición se debería confirmar empíricamente. Tal vez sea este el hecho que diferencia los videojuegos no masivos (non-Internet computerized games, en la terminología del DSM-5) de los MMORPG. El uso adictivo de estos últimos se encuentra en jóvenes y adultos que ya han superado la adolescencia, sugiriendo una complicación de características diferentes con mayor riesgo de cronificarse y de ocasionar consecuencias negativas graves (Carbonell, 2014). 
Independientemente de si nuestro estado actual de conocimientos nos permite utilizar los cuestionarios como instrumentos diagnósticos para detectar adicciones a videojuegos, mensajería, redes sociales, etc. si que emerge una consideración por parte de los propios jóvenes del uso problemático que hacen de las tecnologías de la información y la comunicación. Este hecho invita a una profunda reflexión sobre este tipo de ocio y a la necesidad de que sus cuidadores los consideren como un elemento sobre el que es necesario fomentar un uso saludable. Aunque suene a tópico, son necesarios estudios longitudinales para determinar cómo evoluciona el uso problemático de los videojuegos masivos y no masivos. Se trata de una conducta poco conocida que requiere más investigación.

\section{Agradecimientos}

Esta investigación ha sido posible gracias a la colaboración de los alumnos y profesores de los centros de educación secundaria de Sabadell (IES Ferran Casablancas, IES Arraona, IES Agustí Serra, IES Miquel Crusafont, IES Pau Vila, IES Vallès, IES Jonqueres, IES Ribot i Serra, El Carme, Servator, Bertran, Tarrés, La Immaculada, Mare de Déu de la Salut, Ramar 1, Santa Clara, Sant Nico-lau), de Castellar del Vallès (IES Castellar, IES Puig de la Creu, El Casal, La Immaculada), de Santa Perpètua de Mogoda (IES Estela Ibèrica, IES Rovira Forns, Sagrada Família), de Palau-Solità i Plegamans (Marinada), del IES Sant Quirze del Vallès, del IES Sentmenat y del IES Polinyà, así como de las enfermeras referentes del Programa Salut i Escola: Dolors Alcaraz Sanz, M. Ángeles Gómez Mateo, Concepción Caminal Olivé, Cristina Arranz Delgado, Concepció Mestres Hugas, Piedad Díaz Borja, Mónica Baraut Martínez, María Clotilde Gónzález Calvo, Cecília Quer Raves, Vanessa Cruz Muñoz, Pilar Padilla Monclús, Núria Llistar Verdú, Maria Franquesa Freixanet, Carme Forts Llorens, María José Montoto Lamela, Carmina Gil Guitart, Laura Cubinsà Esquius, Meritxell Virgós Soler, Matilde Fernández Soler, Ángeles Vara Ortiz y Assumpta Fatjó Gené.

Todos ellos participaron en la recogida de datos de este estudio.

Damos las gracias también a Fernando Rupérez Vielba y Marta Serra Laguarta (Servei d'Atenció Primària VallèsOccidental) por sus aportaciones en la elaboración del protocolo; a Cristina Moreno Ramos (Direcció d'Atenció Primària Metropolitana Nord), a Eulàlia Picas Riera, a Josep Arnau Figueras, a Rosa M. Perarnau Piñero y a Gemma Morales Puig (Departament d'Ensenyament - Serveis Territorialsdel Vallès Occidental) y a Paqui Vargas Manzano (Direcció d'Atenció Primària Metropolitana Nord) por su apoyo logístico y su dedicación. Realizada en parte gracias a una ayuda de la Fundación Mapfre 2013.

\section{Conflicto de intereses}

Los autores declaran no tener ningún conflicto de intereses.

\section{Referencias}

Anderson, C. A., Shibuya, A., Ihori, N., Swing, E. L., Bushman, B. J., Sakamoto, A., y Saleen, M. (2010). Violent video game effects on aggres-sion, empathy, and prosocial behavior in eastern and western countries: A meta-analytic review. Psychological Bulletin, 136, 151-173.

Bentler, P.M., y Wu, E.J.C. (2002). EQS 6 for windows. User's guide. Encino, CA: Multivariate Software.

Beranuy, M., Chamarro, A., Graner, C., y Carbonell, X. (2009). Validación de dos escalas breves para evaluar la adicción a Internet y el abuso de móvil. Psicothema, 21, 480-485.

Brown, T.A. (2006). Confirmatory factor analysis for applied research. New York: The Guilford Press.

Carbonell, X. (2014). La adicción a los videojuegos en el DSM-5. Adicciones 26, 91-95.

Carbonell, X., Fúster, H., Chamarro, A., y Oberst, U. (2012). Adicción a Internet y a móvil: Una revisión de estudios empíricos españoles. Papeles del psicólogo, 33, 82-89.

Carbonell, X., Talarn, A., Beranuy, M., Oberst, U., y Graner, C. (2009) Cuando jugar se convierte en un problema: el juego patológico y la adicción a los juegos de rol online. Aloma, 25, 201-220.

Carnagey, N. L., Anderson, C. A., y Bushman, B. J. (2007). The effect of video game violence on physiological desensitization to real-life violence. Journal of Experimental Social Psychology, 43, 489-496. doi:10.1016/j. jesp.2006.05.003

Charlton, J.P., y Danforth, I.D.W. (2007). Distinguishing addiction and high engagement in the context of online game playing. Computers in Human Behavior, 23(3), 1531-1548. doi:10.1016/j.chb.2005.07.002

De la Torre-Luque, A., y Valero-Aguayo, L. (2013). Factores moduladores de la respuesta agresiva tras la exposición a videojuegos violentos. Anales de Psicología, 29(2), 311318. doi:10.6018/analesps.29.2.132071

Estallo, J. A., Masferrer, M. C., y Aguirre, C. (2001). Efectos a largo plazo del uso de videojuegos. Apuntes de Psicología, 19, 161-174.

Ewing, J.A. (1984). Detecting alcoholism. The CAGE questionnaire. JAMA, 252(14), 1905-1907.

Fúster, H., Oberst, U., Griffiths, M. D., Carbonell, X., Chamarro, A., y Talarn, A. (2012). Psychological motivation in online role-playing games: A study of Spanish World of Warcraft players. Anales de Psicología, 28, 274280.

Fúster, H., Chamarro, A., Carbonell, X., y Vallerand, R.J. (2014). Relationship between Passion and Motivation for Gaming in Massively Multiplayer Online Role-Playing Games. Cyberpsychology, Behavior, E̋ Social Networking, 17, 292-297. doi: 10.1089/cyber.2013.0349

Gentile, D.A., Choo, H., Liau, A., Sim, T., y Dongdong Li, D. (2011). Pathological video game use among youths: a two-year longitudinal study. Pediatrics, 127, e319-329. doi: $10.1542 /$ peds.2010-1353 
Haagsma, M.C., Caplan, S.E., Peters, O., y Pieterse, M.E. (2013). A cognitive-behavioral model of problematic online gaming in adolescents aged 12-22 years. Computers in Human Behavior, 29, 202-209. Doi:10.1016/j. chb.2012.08.006

Hu, L., y Bentler, P.M. (1999). Cut off criteria for fit indexes in covariance structure analysis: Conventional criteria versus new alternatives. Structural Equation Modeling, 6, 1-55. doi:10.1080/10705519909540118

King, D. L., Delfabbro, P. H., y Griffiths, M. D. (2013). Trajectories of problem video gaming among adult regular gamers: An 18-month longitudinal study. CyberPsychology $\mathcal{E}$ Behavior, 16, 72-76. doi: 10.1089/cyber.2012.0062.

Kline, R. B. (2010). Principles and practices of structural equation modeling (3rd ed.). New York: The Guilford Press.

Labrador, F., y Villadangos, S. (2010). Menores y nuevas tecnologías: conductas indicadoras de posible problema de adicción. Psicothema, 22, 180-188.

Labrador, F., Villadangos, S., Crespo, M., y Becoña, E. (2013). Desarrollo y validación del cuestionario de uso problemático de nuevas tecnologías (UPNT). Anales de Psicología, 29, 836-847. doi:10.6018/analesps.29.3.159291

Lafrenière, M.C., Vallerand, R.J., Donahue, E.G., y Lavigne, G.L. (2009). On The Costs and Benefits of Gaming: The Role of Passion. CyberPsychology E Behavior, 12, 285290. doi:10.1089/cpb.2008.0234.

Lorenci, M. (2012). Anuario SGAE de las Artes Escénicas, musicales y audiovisuales. Fundación Autor: Madrid.

León, R., y López, M. J. (2003). Los adolescentes y los videojuegos. Apuntes de Psicología, 21, 89-99.

Muñoz-Miralles, R., Ortega-González, R., Batalla-Martínez, C., López-Morón, M.R., Manresa, J.M., y Torán-Monserrat, P. (2014). Acceso y uso de nuevas tecnologías entre los jóvenes de educación secundaria, implicaciones en salud. Estudio JOITIC. Atención Primaria, 46, 77-88. doi: 10.1016/j.aprim.2013.06.001.

Ng, B. D., y Wiemer - Hastings, P. (2005). Addiction to the internet and online gaming. Cyberpsychology $\mathcal{E}^{\circ}$ behavior, 8, 110-113. doi:10.1089/cpb.2005.8.110

Nunnally, J.C. (1978). Psychometric theory (2nd edition). New York: Mc- Graw-Hill.

Oliva, A. (2012). Uso y riesgo de adicciones a las nuevas tecnologías entre adolescentes y jóvenes andaluces. Sevilla: Aguaclara editorial.

Pedrero Pérez, E. J., Rodríguez Monje, M. T., Gallardo Alonso, F., Fernández Girón, M., Pérez López, M., y Chicharro Romero, J. (2007). Validación de un instrumento para la detección de trastornos de control de impulsos y adicciones: el MULTICAGE CAD-4. Trastornos Adictivos, 9, 269-278.

Porter, G., Starcevic, V., Berle, D., y Fenech, P. (2010). Recognizing problem video game use. Australian and New Zealand Journal of Psychiatry, 44, 120-128.
Prot, S., McDonald, K. A., Anderson, C. A., y Gentile, D. A. (2012). Video games: good, bad, or other? Pediatric clinics of North America, 59, 647-658. doi:10.1016/j. pcl.2012.03.016

Tejeiro, R., y Bersabé, R. M. (2002). Measuring problem video game playing in adolescents. Addiction, 97, 16011606. 


\section{Anexo 1. La versión definitiva del cuestionario CERV}

\section{Cuestionario CERV}

A continuación encontrarás algunas afirmaciones sobre tu uso de los videojuegos. Lee atentamente cada frase e indica la frecuencia. Señala la respuesta que más se aproxime a tu realidad.

\begin{tabular}{|c|c|c|c|c|}
\hline & $\begin{array}{c}\text { Nunca/ } \\
\text { Casi } \\
\text { nunca }\end{array}$ & $\begin{array}{l}\text { Algunas } \\
\text { veces }\end{array}$ & $\begin{array}{l}\text { Bastantes } \\
\text { veces }\end{array}$ & $\begin{array}{l}\text { Casi } \\
\text { siempre }\end{array}$ \\
\hline 1. ¿Hasta qué punto te sientes inquieto por temas relacionados con los videojuegos? & a & b & c & $d$ \\
\hline 2. ¿Cuando te aburres, usas los videojuegos como una forma de distracción? & a & $\mathrm{b}$ & c & $d$ \\
\hline $\begin{array}{l}\text { 3. ¿Con que frecuencia abandonas lo que estas haciendo para estar más tiempo jugando a } \\
\text { videojuegos? }\end{array}$ & a & b & c & $d$ \\
\hline $\begin{array}{l}\text { 4. ¿Te han criticado tus amigos o familiares por invertir demasiado tiempo y dinero en los } \\
\text { videojuegos o te han dicho que tienes un problema, aunque creas que no es cierto? }\end{array}$ & $a$ & $b$ & c & $d$ \\
\hline $\begin{array}{l}\text { 5. ¿Has tenido el riesgo de perder una relación importante, un trabajo o una oportunidad } \\
\text { académica por el uso de los videojuegos? }\end{array}$ & $a$ & $\mathrm{~b}$ & c & $d$ \\
\hline $\begin{array}{l}\text { 6. ¿Piensas que tu rendimiento académico se ha visto afectado negativamente por el uso de } \\
\text { los videojuegos? }\end{array}$ & $a$ & $\mathrm{~b}$ & c & $d$ \\
\hline $\begin{array}{l}\text { 7. ¿Mientes a tus familiares o amigos con respecto a la frecuencia y duración del tiempo que } \\
\text { inviertes en los videojuegos? }\end{array}$ & $a$ & $b$ & c & $d$ \\
\hline 8. ¿Cuando tienes problemas, usar los videojuegos te ayuda a evadirte? & a & b & c & $d$ \\
\hline $\begin{array}{l}\text { 9. ¿Con qué frecuencia bloqueas los pensamientos molestos sobre tu vida y los sustituyes por } \\
\text { pensamientos agradables de los videojuegos? }\end{array}$ & $a$ & $b$ & c & $d$ \\
\hline 10. ¿Piensas que la vida sin videojuegos es aburrida, vacía y triste? & a & b & c & d \\
\hline 11. ¿Te enfadas o te irritas, cuando alguien te molesta mientras juegas con algún videojuego? & a & b & c & $d$ \\
\hline 12. ¿Sufres alteraciones de sueño debido a aspectos relacionados con los videojuegos? & a & b & c & $d$ \\
\hline 13. ¿Cuando no juegas con videojuegos te sientes agitado o preocupado? & a & b & c & $d$ \\
\hline $\begin{array}{l}\text { 14. ¿Sientes la necesidad de invertir cada vez más tiempo en los videojuegos para sentirte } \\
\text { satisfecho? }\end{array}$ & a & b & c & $d$ \\
\hline 15. ¿Quitas importancia al tiempo que has estado jugando con videojuegos? & a & b & c & $d$ \\
\hline 16. ¿Dejas de salir con tus amigos para pasar más tiempo jugando con videojuegos? & a & $\mathrm{b}$ & c & $d$ \\
\hline 17. ¿Cuando utilizas los videojuegos, te pasa el tiempo sin darte cuenta? & a & $\mathrm{b}$ & c & $d$ \\
\hline
\end{tabular}

